\title{
Treatment of Streptococcus pneumoniae otitis media in a chinchilla model by transtympanic delivery of antibiotics
}

\author{
Rong Yang, ${ }^{1}$ Vishakha Sabharwal, ${ }^{2}$ Nadya Shlykova, ${ }^{2}$ Obiajulu S. Okonkwo, ${ }^{1}$ Stephen I. Pelton, ${ }^{2}$ \\ and Daniel S. Kohane' \\ 'Laboratory for Biomaterials and Drug Delivery, Department of Anesthesiology, Division of Critical Care Medicine, Children's \\ Hospital Boston, Harvard Medical School, Boston Massachusetts, USA. 'Division of Pediatric Infectious Diseases, Maxwell \\ Finland Laboratory for Infectious Diseases, Boston Medical Center, Boston, Massachusetts, USA.
}

\begin{abstract}
Otits media (OM) is the most frequent indication for antimicrobial prescription to US children. Streptococcus pneumoniae (S. pneumoniae) remains one of the most common pathogens causing $\mathrm{OM}$. Successful eradication of $S$. pneumoniae in the middle ear can be achieved by adhering to a 7-10 day regimen of oral antibiotics. However, oral drug administration is challenging for parents. Lack of adherence has been associated with treatment failure or early relapse. To overcome this challenge, we used a noninvasive formulation to achieve high transtympanic antibiotic flux and cured S. pneumoniae $\mathrm{OM}$ in chinchillas. The formulation consists of a thermosensitive in situ gelling hydrogel, chemical permeation enhancers, and an antibiotic. The direct transport of drugs into the middle ear produced high concentrations of ciprofloxacin (in the range of hundreds of micrograms per milliliter) within the first $\mathbf{2 4}$ hours of administration. Drug concentrations above the minimum inhibitory concentration (MIC) for S. pneumoniae were sustained throughout the 7-day treatment. S. pneumoniae $\mathrm{OM}$ in a chinchilla model was successfully eradicated, without causing tissue toxicity. Transtympanic delivery minimized systemic drug exposure, as evidenced by undetectable levels in blood, measured by high-performance liquid chromatography.
\end{abstract}

Conflict of interest: The authors have declared that no conflict of interest exists.

Submitted: July 6, 2018

Accepted: August 29, 2018

Published: October 4, 2018

Reference information: JCI Insight. 2018;3(19): e123415. https://doi.org/10.1172/jici. insight.123415.

\section{Introduction}

Otitis media (OM) remains a significant health burden for children, despite the introduction of OM guidelines and influenza vaccination $(1,2)$. Over $95 \%$ of children in the US experience at least 1 episode of $\mathrm{OM}$, and $40 \%$ experience more than 4 episodes by the age of 7 , rendering OM the most frequent pediatric disease for which antibiotics are prescribed (3-6). Recurrent OM has been associated with a reduced quality of life for children and their families (7). Moreover, globally, OM leads to more than 25,000 deaths annually, owing to intracranial complications resulting from extension of $\mathrm{OM}$ in the absence of effective treatment, primarily in low-income countries (8).

Currently a 7- to 10-day course of broad spectrum oral antibiotics is the standard of care in young children (1). While recent American Academy of Pediatrics guidelines suggest that $\mathrm{OM}$ in older children can be managed with observation, the guidelines support that $\mathrm{OM}$ in infants is best managed with antibiotic therapy (1). As a result, OM accounts for $21 \%$ of all the antibiotic prescriptions written to US children, representing the primary cause for systemic antibiotic exposure in infancy (9).

Systemic antibiotic treatment can cause harmful side effects such as rashes, vomiting, and diarrhea (10). Recent research has also associated early exposure to antibiotics with health issues (such as obesity and asthma) later in life $(11,12)$. Moreover, the widespread use of systemic antibiotics for a disease of such high prevalence is believed to be responsible, at least in part, for the ongoing increase in antibiotic resistance among nasopharyngeal pathobionts (13). Drug resistance can also result from incomplete adherence to the current multidose, multiday regimens, which is common, especially in young children (14). According to the Centers for Disease Control and Prevention (CDC), 23,000 death and 2 million illnesses in the US are associated with antibiotic-resistant infections, which are difficult to treat (15).

The delivery of antibiotics directly across the tympanic membrane (TM) for local treatment of OM could obviate the concern with systemic antibiotic exposure. However, the TM represents an impermeable 
barrier to virtually all molecules (16), despite its thinness (human TMs are $10-\mu \mathrm{m}$ to $100-\mu \mathrm{m}$ thick) (17). The low permeability of the TM is mostly due to its outermost layer, the stratum corneum, which blocks all but the smallest lipophilic molecules (18). We have used chemical permeation enhancers (CPEs) to reversibly enhance transtympanic delivery of ciprofloxacin, a broad-spectrum fluoroquinolone currently used for OM when tympanostomy tubes are in place (19). The antibiotic and CPEs were loaded into a hydrogel with reverse-thermal gelation properties, designed to gel rapidly upon contacting the warm TM following application into the external canal in young patients. We previously demonstrated that a single dose of this formulation is sufficient to eradicate OM caused by nontypeable Haemophilus influenzae (NTHi) (19).

Streptococcus pneumoniae (S. pneumoniae) accounts for 30\%-50\% of all OM cases, translating to approximately 300 million OM cases worldwide annually (20). Reduced susceptibility or resistance to $\beta$-lactam and macrolide antibiotics is prevalent among isolates of $S$. pneumoniae $(21,22)$. The minimum inhibitory concentration (MIC) of S. pneumoniae is at least 50 times greater than that of NTHi (the MIC of ciprofloxacin against certain strains of $S$. pneumoniae is $0.5-4 \mu \mathrm{g} / \mathrm{ml}$, compared with less than $0.01 \mu \mathrm{g} / \mathrm{ml}$ for NTHi; refs. 23-26). To eradicate $S$. pneumoniae OM, it is crucial to maintain high drug concentrations in the middle ear throughout treatment, which currently requires adherence to a regimen of multiple doses of antibiotics per day over 7-10 days (27). Adhering to this rigorous regimen of outpatient antimicrobial therapy in young children remains challenging. As a result, treatments are often terminated once symptoms are resolved, although the pathogens may not be eradicated. This premature termination of treatment can lead to recurrent $\mathrm{OM}$ and antibiotic resistance.

Here, we studied whether transtympanic drug delivery could be used to eradicate $S$. pneumoniae in a chinchilla model of OM, despite its high MIC.

\section{Results}

\section{Nomenclature}

The hydrogel-based formulation contained 3 components: ciprofloxacin hydrochloride (Cip) at 1\% (w/v) or $4 \%(\mathrm{w} / \mathrm{v})$, a combination of $3 \mathrm{CPEs}(3 \mathrm{CPE})$, and a temperature-responsive hydrogel made of poloxamer 407-polybutylphosphoester (P407-PBP) at $12 \%(\mathrm{w} / \mathrm{v})$. The formulations are referred to as $\mathrm{x} \% \mathrm{Cip}-3 \mathrm{CPE}-$ [P407-PBP], where $\mathrm{x}=1$ or $4 \%(\mathrm{w} / \mathrm{v})$ of ciprofloxacin. Unless specified otherwise, 3CPE denotes a combination of $2 \% \mathrm{w} / \mathrm{v}$ limonene, $1 \% \mathrm{w} / \mathrm{v}$ sodium dodecyl sulfate, and $0.5 \% \mathrm{w} / \mathrm{v}$ bupivacaine hydrochloride. The concentration of P407-PBP was always $12 \% \mathrm{w} / \mathrm{v}$. If a component was absent from a formulation, it was omitted from the above nomenclature. The combination of $3 \mathrm{CPE}$ and concentration of P407-PBP were adopted from our previous report (19).

\section{Synthesis of thermos-sensitive hydrogel}

P407-PBP was synthesized as reported previously (19). The successful addition of PBP groups to the P407 backbone was evidenced by the $\mathrm{P}-\mathrm{O}$ stretch introduced in the spectrum by Fourier transform infrared spectroscopy (FTIR, Supplemental Figure 1; supplemental material available online with this article; https:// doi.org/10.1172/jci.insight.123415DS1). The degree of polymerization (DP) of the PBP groups was about 5 , as calculated using the ratio of the peak areas at $0.90 \mathrm{ppm}$ (the n-butyl side chain in PBP) and $1.14 \mathrm{ppm}$ (P407 backbone) in their spectra by nuclear magnetic resonance (NMR, Supplemental Figure 2).

\section{Improving efficacy of the hydrogel formulation}

The formulation 1\%Cip-3CPE-[P407-PBP] had cured 100\% of animals when applied to the TMs of chinchillas infected with NTHi in our previous work (19) but cured only 1 of 4 chinchillas infected with $S$. pneumoniae (Figure 1). The reduced efficacy reflected the higher MIC for $S$. pneumoniae $(0.5-4 \mu \mathrm{g} / \mathrm{ml})(23$, 24) compared with that of NTHi (less than $0.01 \mu \mathrm{g} / \mathrm{ml})(25,26)$.

The most straightforward way to improve efficacy was to increase the concentration gradient (a major determinant of flux) of ciprofloxacin across the TM. We achieved this by maximizing the loading of Cip in the formulation to $4 \% \mathrm{w} / \mathrm{v}$, which is its water solubility limit at $\mathrm{pH} 3.6$ that we determined experimentally.

The effect of drug concentration on transtympanic permeation kinetics was studied by (a) in vitro diffusion from the bulk polymer matrix and (b) ex vivo permeation across the TM.

In vitro drug release from the hydrogel formulation. In vitro drug release studies showed that the polymer P407-PBP slowed drug release compared with the free drug solution (Supplemental Figure 3). Incorporation 


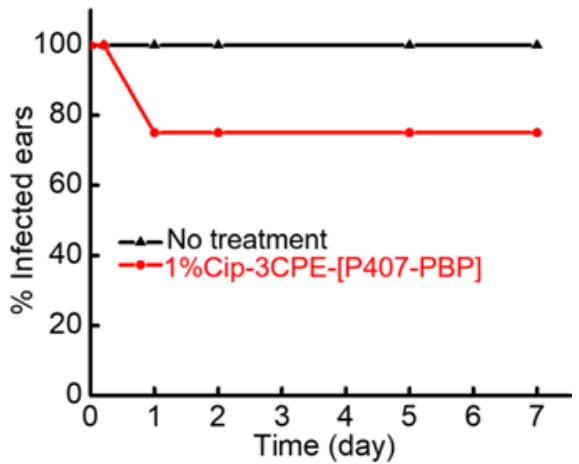

Figure 1. Efficacy of a $1 \%(w / v)$ Ciprofloxacin hydrogel against S. Pneumoniae. A formulation containing $1 \%(\mathrm{w} / \mathrm{v})$ ciprofloxacin, $2 \%(w / v)$ limonene, $1 \%(w / v)$ sodium dodecyl sulfate, $0.5 \%$ $(\mathrm{w} / \mathrm{v})$ bupivacaine hydrochloride, and $12 \%(\mathrm{w} / \mathrm{v})$ poloxamer 407-polybutylphosphoester (1\%Cip-3CPE-[P407-PBP]) was used for the treatment of S. pneumoniae otitis media (OM). Efficacy was indicated by the percentage of animals with OM before (day 0$)$ and after receiving $1 \%$ Cip-3CPE-[P407-PBP] $(n=4)$ and was compared with that of no treatment $(n=4)$. Day 0 reflects status immediately prior to administration of therapeutics.

of CPEs further reduced release. After 48 hours, $5.4 \mathrm{mg}$ of ciprofloxacin (67.5\% of total drug loading) was released from the gel 4\%Cip-3CPE-[P407-PBP]; whereas $1.4 \mathrm{mg}$ of ciprofloxacin (70\% of total drug loading) was released from $1 \%$ Cip-3CPE-[P407-PBP]. The amount of ciprofloxacin released was proportional to initial drug loading, while the percentage of total drug released (e.g., $67.5 \%$ and $70 \%$ after 48 hours for 4\%Cip-3CPE-[P407-PBP] and 1\%Cip-3CPE-[P407-PBP], respectively) remained unchanged.

Ex vivo transtympanic permeation kinetics of the hydrogel formulation. Ex vivo experiments evaluated the rate of drug transport across healthy TMs excised from chinchillas. Hydrogel formulations were placed on the TMs inside auditory bullae harvested from euthanized healthy chinchillas. The bullae were then placed in 12-well plates filled with $3 \mathrm{ml}$ phosphate buffered saline (PBS). At each time point (Figure 2), the drug concentration in the PBS buffer was measured by HPLC, and the bullae were placed into fresh PBS buffer to maintain infinite sink conditions.

In the absence of CPEs, the 4\%Cip-[P407-PBP] polymer matrix decreased transtympanic flux of ciprofloxacin compared with free drug, from $0.37 \mathrm{mg}(0.22-0.68 \mathrm{mg})$ to $0.07 \mathrm{mg}(0.05-0.08 \mathrm{mg})$, although this decrease was statistically insignificant ( $P=0.03$ by Mann-Whitney $U$ test $[\alpha=0.0125]$. Flux data are described with medians, with 25 th and 75 th percentiles in parentheses.). The effect of the polymer matrix was counteracted by the incorporation of 3CPE, increasing flux 5.7-fold to $0.40 \mathrm{mg}(0.33-0.42 \mathrm{mg})$ for $4 \%$ Cip-3CPE-[P407-PBP], ( $P=0.006$ by Mann-Whitney $U$ test, $\alpha=0.0125$ ). The transtympanic flux of $4 \%$ Cip-3CPE-[P407-PBP] was not statistically significantly different from that of $4 \%$ Cip-3CPE free solution $[0.68 \mathrm{mg}(0.46-0.92 \mathrm{mg}), P=0.07$ by Mann-Whitney $U$ test, $\alpha=0.0125$ ). Note that, although the gel reduces flux across the TM, the flux is still more than adequate for therapeutic effect (see below) and the gel is needed to keep the antibiotic on the TM (19). The ciprofloxacin flux observed with 4\%Cip-3CPE-PBP was $\sim 3.5$-fold greater than that reported with $1 \%$ Cip3CPE-[P407-PBP] (0.12 [0.11-0.14] mg, $P=0.006$ by Mann-Whitney $U$ test, $\alpha=0.0125$; ref. 19].

Rheology of the hydrogel formulation. The reverse thermal gelation characteristics of the P407-PBP gels were the key to achieving a formulation that could flow easily onto the warm TM and then gel quickly and firmly. Since gel components can affect gelation properties $(28,29)$, we used shear rheology to confirm that increasing ciprofloxacin loading by a factor of 4 did not affect gelation (Figure 3). The gelation temperature (when the storage modulus exceeded the loss modulus) was $23^{\circ} \mathrm{C}$ at both ciprofloxacin concentrations. At body temperature, the storage and loss moduli of $4 \% \mathrm{Cip}-3 \mathrm{CPE}-[\mathrm{P} 407-\mathrm{PBP}]$ were $\sim 2.2 \mathrm{kPa}$ and $0.1 \mathrm{kPa}$, at which the gel behaved as a solid.

Figure 2. Ex vivo transfer of ciprofloxacin (Cip) across the tympanic membrane (TM) into a receiving chamber. Data are medians with interquartile ranges, $n=4$ for the aqueous solution containing $4 \%(w / v)$ Cip ( $4 \%$ Cip), aqueous solution containing $4 \%$ $(w / v)$ Cip, $2 \%(w / v)$ limonene, $1 \%(w / v)$ sodium dodecyl sulfate, $0.5 \%(w / v)$ bupivacaine hydrochloride (4\%Cip-3CPE), and the formulation containing $4 \%(\mathrm{w} / \mathrm{v})$ Cip and $12 \%(\mathrm{w} / \mathrm{v})$ poloxamer 407-polybutylphosphoester (4\%Cip- [P407-PBP]); $n=7$ for the formulation containing $4 \%(w / v)$ Cip, $2 \%(w / v)$ limonene, $1 \%$ $(\mathrm{w} / \mathrm{v})$ sodium dodecyl sulfate, $0.5 \%(\mathrm{w} / \mathrm{v})$ bupivacaine hydrochloride, and $12 \%(\mathrm{w} / \mathrm{v})$ poloxamer 407 -polybutylphosphoester (4\%Cip-3CPE-[P407-PBP]). Groups were compared with the Mann-Whitney $U$ test; ${ }^{*} P<0.05$; please see text for discussion.

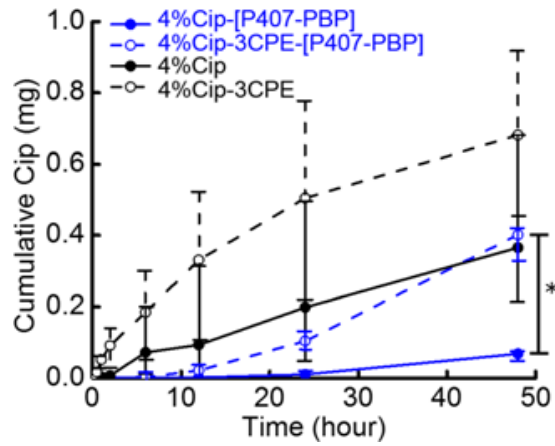




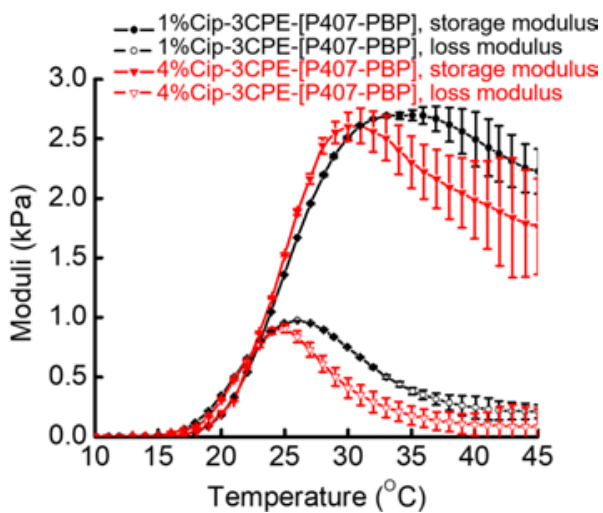

Figure 3. Rheology of formulations. Gelation of the aqueous solution containing $1 \%(w / v)$ ciprofloxacin, $2 \%(w / v)$ limonene, $1 \%(\mathrm{w} / \mathrm{v})$ sodium dodecyl sulfate, $0.5 \%(\mathrm{w} / \mathrm{v})$ bupivacaine hydrochloride, and $12 \%(\mathrm{w} / \mathrm{v})$ poloxamer 407 -polybutylphosphoester (1\%Cip-3CPE-[P407-PBP]) and the solution containing $4 \%(w / v)$ ciprofloxacin, $2 \%(w / v)$ limonene, $1 \%$ $(\mathrm{w} / \mathrm{v})$ sodium dodecyl sulfate, $0.5 \%(\mathrm{w} / \mathrm{v})$ bupivacaine hydrochloride, and $12 \%(\mathrm{w} / \mathrm{v})$ poloxamer 407 -polybutylphosphoester (4\%Cip-3CPE-[P407-PBP]), as a function of temperature, was demonstrated using rheology. $n=3$ for both formulations. Data represent mean \pm SD.

In vivo efficacy of the hydrogel formulation. S. pneumoniae-induced OM was established in chinchillas, as reported (30). Please refer to Supplemental Figure 4 for a timeline of the procedure. In brief, S. pneumoniae was inoculated into the nasopharynx of the animals, followed by direct inoculation 24 hours later into their middle ear. Two days after inoculation, middle ear fluid of the animals was cultured under $S$. pneumoniae growth conditions overnight, and the number of CFU was assessed the next morning (3 days after middle ear inoculation). More than $1 \times 10^{4} \mathrm{CFU}$ indicated successful establishment of the infection and would trigger hydrogel treatments described below on that day.

Infected chinchillas were treated with $200 \mu 1$ of test formulations, deposited via a soft catheter directly onto the TM through the outer ear canal. Middle ear fluid was extracted at 5 hours, 1 day, 2 days, 5 days, and 7 days after treatment, for (a) bacterial culture to determine the bacterial count in the middle ear and for (b) HPLC to quantify drug concentrations (see below). A reduction of the bacterial count in the middle ear by $99.9 \%$ (i.e., a 3-log reduction) was considered an indication of cure (30).

Test animals were grouped into 3 groups: no treatment, 4\%Cip-3CPE, and 4\%Cip-3CPE-[P407-PBP]. The untreated animals and animals treated with $4 \%$ Cip-3CPE showed no sign of reduced inflammation of the TM by visual examination. The bacterial counts in middle ear fluid from those animals (i.e., untreated or treated with $4 \% \mathrm{Cip}-3 \mathrm{CPE}$ ) were not reduced at time point during the 7-day treatment (Figure $4 \mathrm{~A}$ ). One ani$\mathrm{mal}$ in the group receiving $4 \% \mathrm{Cip}-3 \mathrm{CPE}$ had to be sacrificed on day 5 due to severe head tilting. In contrast, OM was cured in 11 of 11 animals treated with 4\%Cip-3CPE-[P407-PBP]. In 45\% of those animals, S. pneumoniae was eradicated within 5 hours of treatment. In $81 \%$ of animals, counts of $S$. pneumoniae in the middle ear dropped to zero by 2 days after gel application (Supplemental Figure 5). Symptoms including inflammation and bulging of the TM resolved within 5-24 hours after treatment with 4\%Cip-3CPE-[P407-PBP] began.

Pharmacokinetics of the hydrogel formulation. The rapid cure of $S$. pneumoniae-induced OM by 4\%Cip$3 \mathrm{CPE}-[\mathrm{P} 407-\mathrm{PBP}]$ resulted from the high concentration of ciprofloxacin in the middle ear, both initially and in a sustained fashion throughout the 7-day treatment (Figure $4 \mathrm{~B}$ ).

In animals receiving no treatment or treatment with $4 \%$ Cip-3CPE, ciprofloxacin was undetectable in their middle ear fluid. In animals treated with 1\%Cip-3CPE-[P407-PBP], the average middle ear drug concentration peaked after 24 hours at $\sim 39 \mu \mathrm{g} / \mathrm{ml}$ and fell to $\sim 3 \mu \mathrm{g} / \mathrm{ml}$ after 3 days. (Again, the MIC to ciprofloxacin of $S$. pneumoniae is 0.5 to $4 \mu \mathrm{g} / \mathrm{ml}$; ref. 23, 24). In the 11 animals treated with $4 \%$ Cip-3CPE[P407-PBP], the average middle ear fluid ciprofloxacin concentration peaked after 5 hours at $369 \mu \mathrm{g} / \mathrm{ml}$, at least 90 times the MIC of S. pneumoniae. Note that animals whose middle ears were sterilized were free of middle ear fluid, and middle ear fluid sampling was thus replaced by lavage of the tympanic bullae. Seven days after the administration of 4\%Cip-3CPE-[P407-PBP], the average drug level in the lavage $(81 \mu \mathrm{g} / \mathrm{ml})$ was still many times greater than the MIC of $S$. pneumoniae.

Undetectable systemic drug exposure from the hydrogel formulation. An important potential advantage of transtympanic delivery of antibiotics is that it could achieve high local drug levels with minimal systemic distribution. To investigate the blood levels of ciprofloxacin, plasma samples were collected at predetermined intervals from the transverse sinuses of chinchillas treated with 4\%Cip-3CPE-[P407-PBP] (Table 1). Ciprofloxacin was undetectable by HPLC at any time point throughout the treatment, despite being supratherapeutic in the middle ear. The undetectable level of ciprofloxacin provided strong evidence for low or no systemic exposure of antibiotics. 

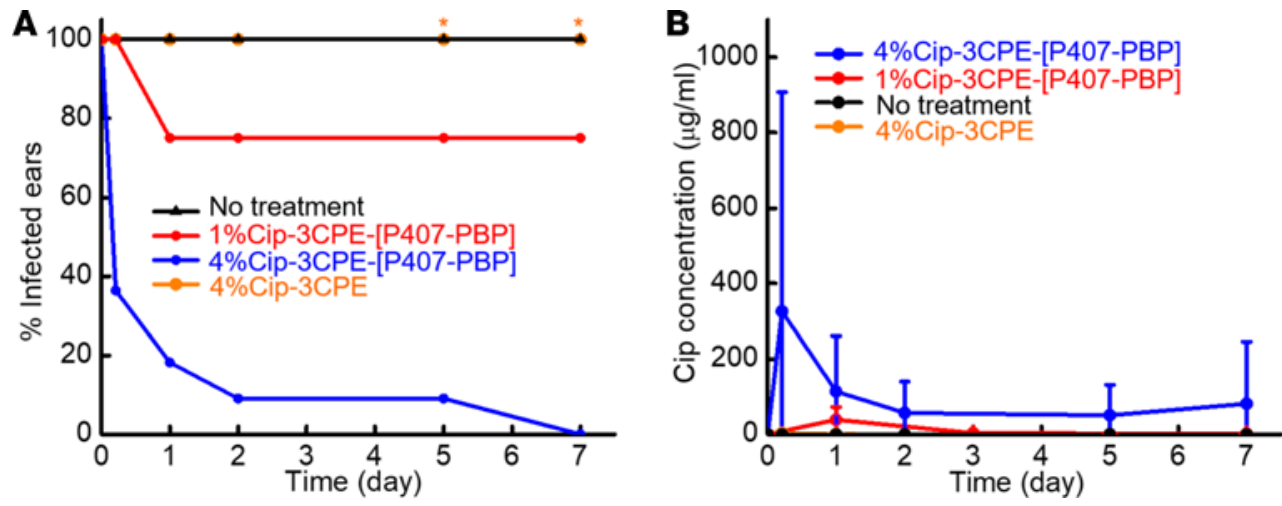

Figure 4. Effect of treatments in S. pneumoniae otitis media (OM). (A) In vivo efficacy (clearance of infection). $n=5$ for the formulation containing $4 \%(\mathrm{w} / \mathrm{v})$ ciprofloxacin, $2 \%(\mathrm{w} / \mathrm{v})$ limonene, $1 \%(\mathrm{w} / \mathrm{v})$ sodium dodecyl sulfate, and $0.5 \%$ $(\mathrm{w} / \mathrm{v})$ bupivacaine hydrochloride (4\%Cip-3CPE); $n=4$ for no treatment and for the formulation containing $1 \%(\mathrm{w} / \mathrm{v})$ ciprofloxacin, $2 \%(\mathrm{w} / \mathrm{v})$ limonene, $1 \%(\mathrm{w} / \mathrm{v})$ sodium dodecyl sulfate, $0.5 \%(\mathrm{w} / \mathrm{v})$ bupivacaine hydrochloride, and $12 \%$ $(\mathrm{w} / \mathrm{v})$ poloxamer 407-polybutylphosphoester (1\%Cip-3CPE-[P407-PBP]); and $n=11$ for the formulation containing $4 \%$ $(\mathrm{w} / \mathrm{v})$ ciprofloxacin, $2 \%(\mathrm{w} / \mathrm{v})$ limonene, $1 \%(\mathrm{w} / \mathrm{v})$ sodium dodecyl sulfate, $0.5 \%(\mathrm{w} / \mathrm{v})$ bupivacaine hydrochloride, and $12 \%(\mathrm{w} / \mathrm{v})$ poloxamer 407-polybutylphosphoester (4\%Cip-3CPE-[P407-PBP]). Asterisk represents 1 animal in group receiving $4 \%$ Cip-3CPE that had to be sacrificed on day 5. (B) The concentration of Cip over time in the middle ear fluid of the same animals as in $\mathbf{A}$. Data represent mean \pm SD.

In vivo biocompatibility in the ear of the hydrogel formulation. Tissue reaction to 4\%Cip-3CPE-[P407-PBP] in the outer ear was benign. By light microscopy (Figure 5), H\&E-stained sections of healthy TMs treated with the gel for 7 days (thickness $9 \pm 3 \mu \mathrm{m}$ ) looked very similar to those from pristine TMs (thickness $10 \pm$ $4 \mu \mathrm{m}, P=0.85$ by Student's $t$ test, $\alpha=0.0125$ ). No inflammation, necrosis, or tissue damage was observed. Without the gel treatment, infected TMs were much thicker $(105 \pm 16 \mu \mathrm{m})$ than healthy TM $\left(P=1 \times 10^{-5}\right.$ by Student's $t$ test, $\alpha=0.0125)$ due to inflammation. After 7 days of treatment with the gel, the TM thickness was reduced to $65 \pm 8 \mu \mathrm{m}(P=0.002$ compared with untreated TM with OM by Student's $t$ test, $\alpha=0.0125)$.

\section{Discussion}

We have achieved very high peak concentrations of ciprofloxacin, in the range of hundreds of micrograms per milliliter, in the middle ear of animals using an ototopical formulation. A single application sustained the therapeutic drug level over the course of the 7-day treatment. The formulation achieved greater than $99.9 \%$ reductions in the middle ear bacterial count in all animals infected with $S$. pneumoniae. This notable efficacy was achieved by increasing the drug loading to the solubility limit and delivering the drug across intact TM in the presence of $3 \mathrm{CPE}$.

We have previously demonstrated that a formulation containing $1 \%$ ciprofloxacin, $3 \mathrm{CPE}$, and $12 \%$ P407-PBP could eradicate the infection of NTHi. Nonetheless, the higher MIC for S. pneumonia prevented the sterilization of the middle ear by this formulation. $S$. pneumoniae has an MIC $(0.5-4 \mu \mathrm{g} / \mathrm{ml}$; that is more

Table 1. Systemic exposure to ciprofloxacin

\begin{tabular}{cc}
\hline Time & Concentration of ciprofloxacin in plasma $(\boldsymbol{\mu g})$ \\
\hline $\mathbf{5}$ hours & Not detected \\
\hline $\mathbf{1}$ day & Not detected \\
\hline $\mathbf{2}$ days & Not detected \\
\hline $\mathbf{5}$ days & Not detected \\
\hline $\mathbf{7}$ days & Not detected \\
\hline
\end{tabular}

Animals were treated with $4 \%(w / v)$ ciprofloxacin, $2 \%(w / v)$ limonene, $1 \%(w / v)$ sodium dodecyl sulfate, $0.5 \%(w / v)$ bupivacaine hydrochloride, and $12 \%(\mathrm{w} / \mathrm{v}$ ) poloxamer 407 -polybutylphosphoester (4\%Cip-3CPE-[P407-PBP]). Blood samples were analyzed for ciprofloxacin content by HPLC at predetermined intervals after gel application $(n=11)$. The limit of detection for ciprofloxacin using HPLC is about $1 \mathrm{ng} / \mathrm{ml}$. 


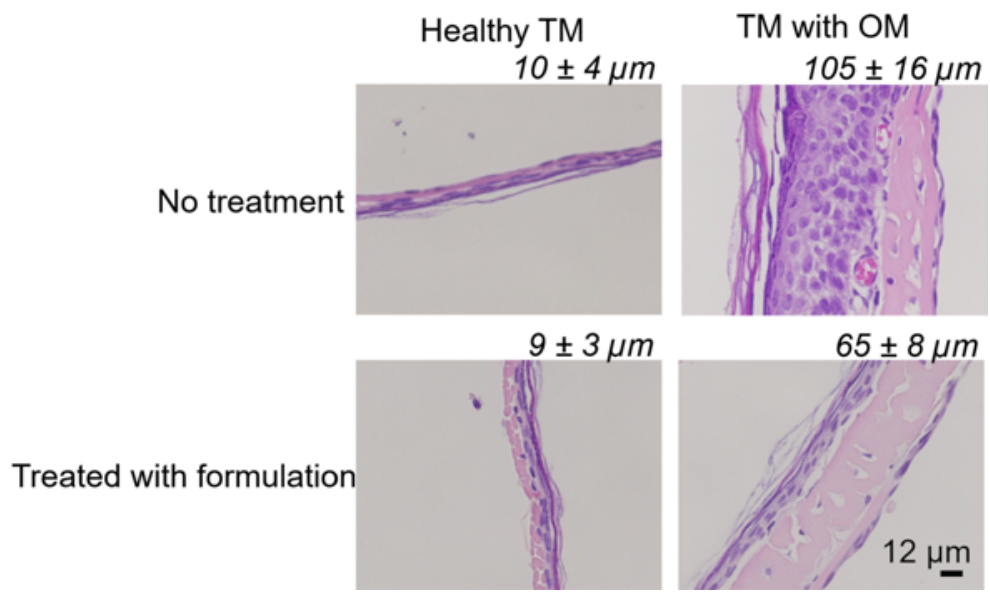

Figure 5. (Representative H\&E-stained sections of tympanic membranes (TM). The H\&E-stained sections were obtained from healthy TMs and of TMs after 7 days of otitis media (OM), without or after treatment with the formulation containing 4\% (w/v) ciprofloxacin, $2 \%(w / v)$ limonene, $1 \%(w / v)$ sodium dodecyl sulfate, $0.5 \%(w / v)$ bupivacaine hydrochloride, and $12 \%(w / v)$ poloxamer 407-polybutylphosphoester (4\%Cip-3CPE-[P407-PBP]). $n=11$ for healthy TM with no treatment; $n=4$ for TM with OM with no treatment; $n=4$ for healthy TM with treatment; $n=11$ for TM with OM with treatment. Scale bar: $12 \mu \mathrm{m}$. The average thickness of TMs ( \pm SD) for each group is shown above their respective panels.

than 50 times greater than that of $N T H i[<0.01 \mu \mathrm{g} / \mathrm{ml}]$; refs. 23-26). Moreover, the successful eradication of $S$. pneumoniae $\mathrm{OM}$ requires a peak middle ear drug concentration that is more than 10 times greater than the $\operatorname{MIC}(27,31)$.

We adopted a straightforward solution to this problem, increasing drug loading in the hydrogel formulation. The adapted formulation was proven effective and achieved pharmacokinetics more favorable to oral antibiotics, as well as a faster resolution of the symptoms.

The amount of ciprofloxacin released under infinite sink condition was proportional to the initial drug loading (i.e., the percentage of drug release remained unchanged). The linear correlation between drug loading and in vitro drug release implies that the transport of ciprofloxacin inside the polymer matrix was passive diffusion driven by a chemical potential gradient. The transtympanic drug flux was roughly proportional to the drug loading, as well, indicating that the diffusion of drug across the TM is likely simple Fickian diffusion.

Rheology of the hydrogel formulation remained largely unchanged upon increasing ciprofloxacin concentration from $1 \%-4 \%$. There was a minor decrease in the storage modulus $\left(\mathrm{G}^{\prime}\right)$ at $37^{\circ} \mathrm{C}$, which did not result in any meaningful change in the efficacy or biocompatibility of the formulation, as discussed below.

In the in vivo $S$. pneumoniae OM model, the peak drug concentration achieved by 1 dose of hydrogel containing $8 \mathrm{mg}$ ciprofloxacin was nearly a thousand times that of oral antibiotic administration at a dose of $6 \mathrm{mg}$ daily (32). Drug concentration in the middle ear was maintained at tens to hundreds of times the therapeutic level throughout the 7-day treatment. The effectiveness of this favorable pharmacokinetics was manifested by the rapid cure - most animals (81\%) cleared their infections in the first 2 days.

Systemic drug distribution was undetectable by HPLC, despite being supratherapeutic in the middle ear. The formulation thus enables the use of highly efficacious antimicrobial agents where there might be reservations about using them systemically, as is the case with ciprofloxacin in children (33). Note that it is a similar reasoning that allows the use of ciprofloxacin drops in children with myringotomy tubes in their TMs. There was no histological evidence of TM injury after 1 week of contact with the hydrogel. None of the common side effects associated with taking oral antibiotics, such as diarrhea and vomiting, was observed in the animals receiving the local treatment.

The human TM is, on average, approximately 10 times thicker than chinchilla's (17). However, we have achieved a peak middle ear drug concentration $(369 \mu \mathrm{g} / \mathrm{ml})$ that is $92-738$ times the MIC of $S$. pneumoniae $(0.5-4 \mu \mathrm{g} / \mathrm{ml})$. If the thicker TM in humans were to impede efficacy, it might be possible to enhance bactericidal effect with a more potent antibiotic or by enhancing flux further by altering the composition of CPEs (34).

Conclusion. A single ototopical application of 4\%Cip-3CPE-[P407-PBP] hydrogel was able to cure S. pneumoniae $\mathrm{OM}$ in chinchillas. The peak drug concentration in the middle ear was hundreds of micrograms per 
milliliter, almost a thousand times higher than the reported peak drug level achieved by oral antibiotics. Middle ear drug levels were supratherapeutic throughout the 7-day treatment, without side effects or tissue damages.

\section{Methods}

Study design. Objective of the research is to develop a noninvasive, transtympanic drug delivery platform for $S$. pneumoniae OM. Successful treatment of NTHi OM has been demonstrated previously (19). We focused on developing and evaluating formulations in a $S$. pneumoniae OM animal model. The experiments compared the effect of drug loading on TM permeability and $S$. pneumoniae OM cure rate. During ex vivo experiments, data collection was stopped after 48 hours due to microbial growth on harvested TM, whereas during in vivo experiments, data collection was stopped after 7 days because OM would either be cleared or cause the animal severe illness that requires euthanasia. In vivo experiments were blinded. All experiments were randomized.

Materials. COP (2-chloro-2-oxo-1,3,2-dioxaphospholane), 1,8-diazabicyclo[5.4.0]undec-7-ene (DBU), n-butanol, diethyl ether, acetic acid, trimethylamine, anhydrous dichloromethane (DCM), and anhydrous tetrahydrofuran (THF) were used as received from MilliporeSigma. Kolliphor P 407 microprilled (poloxamer 407) was received from BASF.

Synthesis of butoxy-2-oxo-1,3,2-dioxaphospholane (BP). BP was prepared by condensation reaction of COP and n-butanol. COP (5.0 g, $35 \mathrm{mmol})$ in anhydrous THF $(50 \mathrm{ml})$ was added to a stirring solution of n-butanol $(2.6 \mathrm{~g}, 35 \mathrm{mmol})$ and trimethylamine $(3.9 \mathrm{~g}, 39 \mathrm{mmol})$ in anhydrous THF $(100 \mathrm{ml})$ at $0^{\circ} \mathrm{C}$ dropwise. The reaction mixture was stirred in an ice bath for 12 hours upon completed addition. Upon complete conversion of COP, the reaction mixture was filtered and the filtrate was concentrated. The concentrated filtrate was purified by vacuum distillation under reduced vacuum to yield a viscous colorless liquid.

Synthesis of P407-PBP. P407-PBP was synthesized by ring opening polymerization (ROP) of BP with $\mathrm{P} 407$ as the macroinitiator, in the presence of DBU (an organocatalyst) at $-20^{\circ} \mathrm{C}(35) . \mathrm{P} 407(8.1 \mathrm{~g}, 0.56$ $\mathrm{mmol})$ and BP $(1.0 \mathrm{~g}, 5.6 \mathrm{mmol})$ in anhydrous DCM $(0.5 \mathrm{ml})$ were added to a flame-dried Schlenk flask $(10 \mathrm{ml})$ equipped with a stir bar. The reaction mixture was flushed with nitrogen gas for 5 minutes while immersed in an ice bath with saturated $\mathrm{NaCl}$ solution. A solution of DBU in anhydrous DCM $(0.13 \mathrm{~g}, 0.84$ $\mathrm{mmol}$ ) was added to the stirring solution via a syringe dropwise while maintaining the reaction under nitrogen gas atmosphere. Upon completion of the reaction, excess amount of acetic acid in DCM was added to the reaction mixture to quench the reaction. The product was purified by precipitation into ether ( 3 times) and dried under vacuum to obtain a white powder product.

Hydrogel formation. P407-PBP hydrogel formulations were made by adding powdered polymers to aqueous solutions. Gels of varying P407-PBP weight percentage (10\%-18\%) can be prepared by simple dissolution in a cold room to allow for better solubility.

Gelation temperature. Gelation temperature was quantified using linear oscillatory shear rheology measurements $\left(100 \mathrm{rads}^{-1}, 1 \%\right.$ strain, $\left.1^{\circ} \mathrm{C} \mathrm{min}{ }^{-1}\right)$. Gelation temperature was taken as the temperature at which the storage modulus $\left(G^{\prime}\right)$ becomes greater than the loss modulus $\left(G^{\prime \prime}\right)$. The changes of $G^{\prime}$ and $G^{\prime \prime}$ over temperatures ranging from $10^{\circ} \mathrm{C}$ to above body temperature were recorded to reflect changes in mechanical properties.

In vitro release studies. The release of ciprofloxacin from each formulation was measured using a diffusion system. Transwell membrane inserts $\left(0.4 \mu \mathrm{m}\right.$ pore size, $1.1 \mathrm{~cm}^{2}$ area; Costar $)$ and 24-well culture plates were employed as the donor and acceptor chambers, respectively. Each formulation (200 $\mu \mathrm{lof}$ each) was pipetted directly onto prewarmed filter inserts to obtain a solid hydrogel. Filter inserts (donor compartments) with formed gels were suspended in wells (acceptor compartments) filled with prewarmed PBS, and the plates were then incubated in a $37^{\circ} \mathrm{C}$ oven. At each time point $(0.5,1,2,6,12,24$, and 48 hours), $1 \mathrm{ml}$ aliquots of the PBS receiving media were sampled and inserts sequentially moved into a new well with fresh PBS. Aliquots were suspended in 70:30 acetonitrile/PBS to ensure total drug dissolution. Sample aliquots were chromatographically analyzed with HPLC to determine ciprofloxacin concentrations $(\lambda=275 \mathrm{~nm})$. More details regarding the ciprofloxacin measurement and HPLC conditions can be found in ref. 36. Experiments were performed in quadruplicate.

Ex vivo permeation experiment. The cross-TM permeation rate of ciprofloxacin was determined with auditory bullae harvested from healthy chinchillas. All formulations were applied into the bullae kept at $37^{\circ} \mathrm{C}$ and deposited onto the TMs. The volume applied was $200 \mu \mathrm{l}$, which translates to $8 \mathrm{mg}$ ciprofloxacin. Permeation of ciprofloxacin across TM into the receiving chamber was quantified using HPLC. Detailed information regarding TM harvesting, TM electrical resistance measurement, and configuration of the ex vivo permeation experiment can be found in ref. 36 . 
Histopathology. Formulations were administered to the ear canals of live healthy/OM chinchillas. Seven days later, they were euthanized as described elsewhere (36). Following sacrifice, the TMs were excised and immediately fixed in 10\% neutral buffered formalin overnight, then decalcified, embedded in paraffin, sectioned (5- $\mu \mathrm{m}$ thick), and stained with H\&E by the Department of Pathology at Boston Children's Hospital (Boston, Massachusetts, USA; fee for service), using standard techniques. All stained specimens were evaluated under light microscopy (Olympus FSX-100).

S. pneumoniae OM model and pharmacokinetics. All procedures and manipulations were performed using sedation analgesia with a mixture of ketamine and xylazine given intramuscularly in accordance with approved IACUC protocols at Boston University Medical Center. Please refer to Supplemental Figure 4 for a timeline of the procedures below. Baseline plasma samples were obtained through the transverse sinus 24 hours prior to bacterial inoculation. To collect blood through the transverse sinus, hair was removed from the scalp. The denuded spot was cleaned with povidone-iodine and then with $70 \%$ ethanol. A 25-guage needle was then used to pierce the skin in the denuded spot, in between the left and right auditory bullae. The needle only penetrated $1-2 \mathrm{~mm}$ beneath the skin to reach the transverse sinus for blood collection (37).

Isolates of $S$. pneumoniae grown to the mid-log phase $\left(1 \times 10^{6} \mathrm{cfu} / \mathrm{ml}\right)$ were diluted in HBSS and inoculated into the nasopharynx of animals. This procedure was followed by direct inoculation (100 cfu per animal) into the middle ear through the dorsal aspect of the tympanic bullae (i.e., not through the TM). Animals were monitored daily via tympanometry and otoscopy. Once abnormality was identified, the middle ear cavity was accessed 48-72 hours later as described previously (38). The animals were considered infected if the bacterial count in their middle ear fluid cultures was above $1 \times 10^{4} \mathrm{cfu}$. Once the middle ear infection was confirmed, the chinchillas were treated with $200 \mu 1$ test formulations deposited via a soft catheter directly on to the TM through their outer ear canal. Cure was defined as greater than $99.9 \%$ reduction in the bacterial count of the middle ear fluid culture within 7 days after a treatment was given. Middle ear fluid was obtained with a 22-gauge angiocatheter connected to an empty tuberculin syringe, 10-20 $\mu 1$ of middle ear fluid was diluted 1:10 in HBSS, and 3 serial 10-fold dilutions were prepared. The lower limit of detection of viable organisms in middle ear fluid using this dilution series was $100 \mathrm{cfu} \mathrm{ml}^{-1}$. Direct and indirect ear examination was performed every 1-2 days until the middle ear cultures were sterile. Serial plasma samples were obtained during the experiment to determine systemic drug levels.

Statistics. For the ex vivo experiments, a sample size of 4 for each formulation was chosen, which would provide $80 \%$ power to detect $50 \%$ differences in flux based on power analysis using the nonparametric Friedman test (version 7.0, nQuery Advisor, Statistical Solutions). Sample sizes of 4-11 were used for the in vivo experiments, which were supported by our published experience (39). Statistical analysis was conducted using MatLab software (version R2018a, The MathWorks, Inc.). Most data were described with means and SDs and compared by 2 -tailed unpaired Student's $t$ tests. Two-tailed $P<0.05$ with appropriate Bonferroni-Sidak adjustment for multiple comparisons were considered statistically significant in order to control type I error. Therefore, for the TM thickness comparisons (4 comparisons), $\alpha=0.05 / 4=0.0125$, so $P<$ 0.0125 was required for statistical significance. In circumstances where data were not normally distributed, data were presented as medians with 25 th and 75 th percentiles in parentheses. Those data were analyzed with the Mann-Whitney $U$ test.

Study approval. Healthy adult male chinchillas weighting 500-650 g were purchased from Ryerson Chinchilla Ranch (Plymouth, Ohio, USA) and cared for in accordance with protocols approved institutionally and nationally. Experiments were carried out in accordance with the Boston Children's Hospital and Boston University Medical Center Animal Use Guidelines and approved by each institution's Animal Care and Use Committee.

\section{Author contributions}

RY synthesized the polymer and conducted FTIR and NMR. RY and OSO conducted in vitro and ex vivo experiments. RY, VS, OSO, and NS performed in vivo experiments. RY, VS, SIP, and DSK contributed to study design. RY, VS, SIP, and DSK performed data analysis and interpretation. RY, VS, DSK, and SIP drafted the article and/or edited it critically for important intellectual content. 


\section{Acknowledgments}

This work was financially supported by NIHDC015050 to DSK, NIH 1K99 DC016644-01A1 to RY, Trailblazer Research Grant by the Department of Anesthesia at Boston Children's Hospital to RY, Charles H. Hood Foundation Child Health Research Award to RY, the Center for Integration of Medicine and Innovative Technology, and Shereta Seelig Charitable Foundation Trust to VS, and it is supported under US Army Medical Research Acquisition Activity Cooperative Agreement subcontract no. W81XWH-09-2-0001 and Massachusetts General Hospital to SIP.

Address correspondence to: Daniel S. Kohane, Boston Children's Hospital, 300 Longwood Avenue, Enders Research Building, Enders 361, Boston, Massachusetts, 02115 USA. Phone: 617.919.2364; Email: daniel. kohane@childrens.harvard.edu.

1. Lieberthal AS, et al. The diagnosis and management of acute otitis media. Pediatrics. 2013;131(3):e964-e999.

2. American Academy of Pediatrics Subcommittee on Management of Acute Otitis Media. Diagnosis and management of acute otitis media. Pediatrics. 2004;113(5):1451-1465.

3. Berman S. Otitis media in children. N Engl J Med. 1995;332(23):1560-1565.

4. Freid VM, Makuc DM, Rooks RN. Ambulatory health care visits by children: principal diagnosis and place of visit. Vital Health Stat 13. 1998;(137):1-23

5. Todberg T, Koch A, Andersson M, Olsen SF, Lous J, Homøe P. Incidence of otitis media in a contemporary Danish National Birth Cohort. PLoS One. 2014;9(12):e111732.

6. Lanphear BP, Byrd RS, Auinger P, Hall CB. Increasing prevalence of recurrent otitis media among children in the United States. Pediatrics. 1997;99(3):E1.

7. Ryborg CT, Søndergaard J, Lous J, Munck A, Larsen PV, Thomsen JL. Quality of life in children with otitis media--a cohort study. Fam Pract. 2014;31(1):30-37.

8. Monasta L, et al. Burden of disease caused by otitis media: systematic review and global estimates. PLoS One. 2012;7(4):e36226.

9. Finkelstein JA, Metlay JP, Davis RL, Rifas-Shiman SL, Dowell SF, Platt R. Antimicrobial use in defined populations of infants and young children. Arch Pediatr Adolesc Med. 2000;154(4):395-400.

10. Cunha BA. Antibiotic side effects. Med Clin North Am. 2001;85(1):149-185.

11. Risnes KR, Belanger K, Murk W, Bracken MB. Antibiotic exposure by 6 months and asthma and allergy at 6 years: Findings in a cohort of 1,401 US children. Am J Epidemiol. 2011;173(3):310-318.

12. Cox LM, Blaser MJ. Antibiotics in early life and obesity. Nat Rev Endocrinol. 2015;11(3):182-190.

13. Brook I, Gober AE. Antimicrobial resistance in the nasopharyngeal flora of children with acute otitis media and otitis media recurring after amoxicillin therapy. J Med Microbiol. 2005;54(Pt 1):83-85.

14. Zielnik-Jurkiewicz B, Bielicka A. Antibiotic resistance of Streptococcus pneumoniae in children with acute otitis media treatment failure. Int J Pediatr Otorhinolaryngol. 2015;79(12):2129-2133.

15. About Antimicrobial Resistance. Center for Disease Control and Prevention. https://www.cdc.gov/drugresistance/about.html. Updated March 5, 2018. Accessed September 12, 2018.

16. Doyle WJ, Alper CM, Seroky JT, Karnavas WJ. Exchange rates of gases across the tympanic membrane in rhesus monkeys Acta Otolaryngol. 1998;118(4):567-573.

17. Van der Jeught S, Dirckx JJ, Aerts JR, Bradu A, Podoleanu AG, Buytaert JA. Full-field thickness distribution of human tympanic membrane obtained with optical coherence tomography. J Assoc Res Otolaryngol. 2013;14(4):483-494.

18. Yang R, Wei T, Goldberg H, Wang W, Cullion K, Kohane DS. Getting Drugs Across Biological Barriers. Adv Mater Weinheim. 2017;29(37).

19. Yang R, et al. Treatment of otitis media by transtympanic delivery of antibiotics. Sci Transl Med. 2016;8(356):356ra120.

20. Bergenfelz C, Hakansson AP. Streptococcus pneumoniae Otitis Media Pathogenesis and How It Informs Our Understanding of Vaccine Strategies. Curr Otorhinolaryngol Rep. 2017;5(2):115-124.

21. Littorin N, Ahl J, Uddén F, Resman F, Riesbeck K. Reduction of Streptococcus pneumoniae in upper respiratory tract cultures and a decreased incidence of related acute otitis media following introduction of childhood pneumococcal conjugate vaccines in a Swedish county. BMC Infect Dis. 2016;16(1):407.

22. Van Dyke MK, et al. Etiology of Acute Otitis Media in Children Less Than 5 Years of Age: A Pooled Analysis of 10 Similarly Designed Observational Studies. Pediatr Infect Dis J. 2017;36(3):274-281.

23. Kayser FH, Novak J. In vitro activity of ciprofloxacin against gram-positive bacteria. An overview. Am J Med. 1987;82(4A):33-39.

24. Patel SN, et al. Susceptibility of Streptococcus pneumoniae to fluoroquinolones in Canada. Antimicrob Agents Chemother. 2011;55(8):3703-3708

25. Pérez-Vázquez M, Román F, Aracil B, Cantón R, Campos J. In vitro activities of garenoxacin (BMS-284756) against Haemophilus influenzae isolates with different fluoroquinolone susceptibilities. Antimicrob Agents Chemother. 2003;47(11):3539-3541.

26. Hirakata Y, et al. Antimicrobial activities of piperacillin-tazobactam against Haemophilus influenzae isolates, including beta-lactamase-negative ampicillin-resistant and beta-lactamase-positive amoxicillin-clavulanate-resistant isolates, and mutations in their quinolone resistance-determining regions. Antimicrob Agents Chemother. 2009;53(10):4225-4230.

27. Hoberman A, et al. Shortened Antimicrobial Treatment for Acute Otitis Media in Young Children. N Engl J Med. 2016;375(25):2446-2456.

28. Hecht E, Mortensen K, Gradzielski M, Hoffmann H. Interaction of ABA block copolymers with ionic surfactants. Influence on micellization and gelation. J Phys Chem. 1995;99(13):4866-4874. 
29. Wanka G, Hoffmann H, Ulbricht W. Phase Diagrams and Aggregation Behavior of Poly(oxyethylene)-Poly(oxypropylene)Poly(oxyethylene) Triblock Copolymers in Aqueous Solutions. Macromolecules. 1994;27(15):4145-4159.

30. Sabharwal V, Figueira M, Pelton SI, Pettigrew MM. Virulence of Streptococcus pneumoniae serotype 6C in experimental otitis media. Microbes Infect. 2012;14(9):712-718.

31. Levison ME, Levison JH. Pharmacokinetics and pharmacodynamics of antibacterial agents. Infect Dis Clin North Am. 2009;23(4):791-815

32. Lovdahl M, Steury J, Russlie H, Canafax DM. Determination of ciprofloxacin levels in chinchilla middle ear effusion and plasma by high-performance liquid chromatography with fluorescence detection. J Chromatogr. 1993;617(2):329-333.

33. Adefurin A, Sammons H, Jacqz-Aigrain E, Choonara I. Ciprofloxacin safety in paediatrics: a systematic review. Arch Dis Child. 2011;96(9):874-880

34. Yang R, Okonkwo OS, Zurakowski D, Kohane DS. Synergy between chemical permeation enhancers and drug permeation across the tympanic membrane [published online ahead of print June 19, 2018]. J Control Release. https://doi.org/10.1016/j. jconrel.2018.06.019.

35. Iwasaki Y, Yamaguchi E. Synthesis of well-defined thermoresponsive polyphosphoester macroinitiators using organocatalysts Macromolecules. 2010;43(6):2664-2666.

36. Khoo X, et al. Formulations for transtympanic antibiotic delivery. Biomaterials. 2013;34(4):1281-1288.

37. McClure DE. Clinical pathology and sample collection in the laboratory rodent. Vet Clin North Am Exot Anim Pract. 1999;2(3):565-590.

38. Sabharwal V, Ram S, Figueira M, Park IH, Pelton SI. Role of complement in host defense against pneumococcal otitis media Infect Immun. 2009;77(3):1121-1127.

39. Pelton SI, Figueira M, Albut R, Stalker D. Efficacy of linezolid in experimental otitis media. Antimicrob Agents Chemother. 2000;44(3):654-657. 